\title{
eration of Spatial Decision Alternatives on a Planar Subdivision of the Study Area
}

\section{Salem Chakhar and Vincent Mousseau}

LAMSADE, University of Paris Dauphine,

du Maréchal de Lattre de Tassigny, 75775 Paris Cedex 16, France,

\{chakhar, mousseau\}@lamsade. dauphine.fr,

WWW home page: http://www.lamsade.dauphine.fr

ract. Outranking methods, a family of multicriteria analysis tools, better with the ordinal aspects of spatial decision problems. Howit is recognized that these methods are subject to computational ations with respect to the number of alternatives. This paper proan approach to generate these alternatives based on a planar subon of the study area. The planar subdivision is obtained by com$\mathrm{g}$ a set of criteria maps. The result is a set of non-overlapping polyspatial units. Punctual, linear and areal decision alternatives, connally used in spatial multicriteria analysis, are then constructed individual, a collection of linearly adjacent or a collection of conus spatial units. This permits to reduce substantially the number ernatives enabling the use of outranking methods.

words: Planar subdivision, GIS, Multicriteria Analysis, Decision hatives

\section{duction}

a analysis (MCA) tools are often used in spatial contexts to evaluate re a set of potential decision alternatives - often modeled through near or areal entities and evaluated on several criteria - in order to ricted subset for implementation. They have been incorporated into l information systems (GIS) to enhance its modeling and analysis The author in [7] provides a recent review on GIS-MCA integration period 1990-2004. MCA methods are commonly categorized, based of alternatives, into discrete and continuous. There are two families within the discrete category: utility function-based approach and based approach.

MCA based-GIS systems use utility function-based methods (e.g. methods still dominate today and only few works (e.g. [8]) use based ones. Outranking methods cope better with spatial decision nce they: (i) permit to consider qualitative evaluation criteria (in adrantitative ones) for which preference intervals ratios have no sense; to consider evaluation criteria with heterogenous scales that coding 
them into one common scale is very difficult or artificial; (iii) avoid the compensation between evaluation criteria; and (iv) require fewer amount of information from the decision maker (DM). But the major drawback of outranking methods (except those devoted to multicriteria sorting problems) is that they are not suitable for problems implying a large or infinite number of alternatives. Indeed, it is recognized that these methods are subject to computational limitations with respect to the number of alternatives [9] as most methods require pairwise comparison across all alternatives.

In this paper, we propose an approach to generate spatial alternatives based on a planar subdivision, that we call decision map, of the study area. The planar subdivision is obtained by combining a set of criteria maps. The result is a set of non-overlapping polygonal spatial units. Punctual, linear or areal decision alternatives are then constructed as an individual, a collection of linearly adjacent, or a collection of contiguous spatial units. This permits to reduce substantially the number of alternatives enabling the use of outranking methods.

The rest of the paper is as follows. Section 2 provides the background. Section 3 briefly introduces multicriteria analysis. Section 4 introduces the concept of decision map. Section 5 proposes solutions for the generation of spatial decision alternatives. Section 6 briefly presents the developed prototype. Section 7 concludes the paper.

\section{Background}

We consider only simple area, line and point features of $\mathbf{R}^{2}$. In the rest of the paper, the letters $P, L$, and $Q$ are used to indicate point, line, and area features, defined as follows: (i) An area feature $Q$ is a two-dimensional open points-set of $\mathbf{R}^{2}$ with simply connected interior $Q^{\circ}$ (with no hole) and simply connected boundary $\partial Q$; (ii) A line feature $L$ is a closed connected one-dimensional pointsset in $\mathbf{R}^{2}$ with no self-intersections and with two end-points. The boundary $\partial L$ of $L$ is a set containing its two endpoints and its interior $L^{\circ}$ is the set of the other points; and (iii) A point feature $P$ is zero-dimensional set consisting of only one element of $\mathbf{R}^{2}$. The interior $P^{\circ}$ of a point feature $P$ is the point itself and its boundary is empty (i.e. $\partial P=\emptyset$ ). Below, the symbol $\gamma$ may represent anyone of the three feature types.

There are several proposals for classifying topological spatial relationships (see [3] for a comparative study of some classification methods). These classifications are based on the intersection of boundaries, interiors and exteriors of features. In [2] the authors introduce the CBM (Calculus-Based Method) based on object calculus that takes into account the dimension of the intersections. The authors provide formal definitions of five (touch, in, cross, overlap, and disjoint) relationships and for boundary operators. They also proved that these operators are mutually exclusive, and they constitue a full converging of all topological situations. In the following, we recall the definitions of the CBM. 
Definition 1. The touch relationship applies to all groups except point/point one:

$$
\left(\gamma_{1}, \text { touch, } \gamma_{2}\right) \Leftrightarrow\left(\gamma_{1}^{\circ} \cap \gamma_{2}^{\circ}=\emptyset\right) \wedge\left(\gamma_{1} \cap \gamma_{2} \neq \emptyset\right)
$$

Definition 2. The in relationship applies to every group:

$$
\left(\gamma_{1}, \text { in, } \gamma_{2}\right) \Leftrightarrow\left(\gamma_{1} \cap \gamma_{2}=\gamma_{1}\right) \wedge\left(\gamma_{1}^{\circ} \cap \gamma_{2}^{\circ} \neq \emptyset\right)
$$

Definition 3. The cross relationship applies to line/line and line/area groups:

$$
\begin{gathered}
\left(L_{1}, \operatorname{cross}, L_{2}\right) \Leftrightarrow\left(L_{1} \cap L_{2} \neq \emptyset\right) \wedge\left(\operatorname{dim}\left(L_{1} \cap L_{2}\right)=0\right) \\
(L, \operatorname{cross}, Q) \Leftrightarrow(L \cap Q \neq \emptyset) \wedge(L \cap Q \neq L)
\end{gathered}
$$

Definition 4. The overlap relationship applies to area/area and line/line groups:

$$
\begin{gathered}
\left(\gamma_{1} \text {, overlap, } \gamma_{2}\right) \Leftrightarrow\left(\operatorname{dim}\left(\gamma_{1}^{\circ}\right)=\operatorname{dim}\left(\gamma_{2}^{\circ}\right)=\operatorname{dim}\left(\gamma_{1}^{\circ} \cap \gamma_{2}^{\circ}\right)\right) \\
\wedge\left(\gamma_{1} \cap \gamma_{2} \neq \gamma_{1}\right) \wedge\left(\gamma_{1} \cap \gamma_{2} \neq \gamma_{2}\right)
\end{gathered}
$$

Definition 5. The disjoint relationship applies to every group:

$$
\left.\left(\gamma_{1}, \text { disjoint, } \gamma_{2}\right) \Leftrightarrow\left(\gamma_{1} \cap \gamma_{2}=\emptyset\right)\right)
$$

In order to enhance the use of the above relationships, the authors in [2] have defined operators able to extract boundaries from area and lines features. The boundary operator b for an area feature $Q$ returns the circular line of $\partial Q$. The boundary operators $f$ and $t$ for a line feature return the two end-points features of $L$.

\section{Multicriteria analysis}

In MCA the DM has to choose among several possibilities, called alternatives, on the basis of a set of, often conflicting, evaluation criteria. The set of alternatives $A$ may be finite (or denumerable) or infinite. The MCA methods are categorized on basis of set $A$ into discrete and continuous. In this paper we are concerned with the first category. Let $A=\left\{x_{1}, x_{2}, \cdots, x_{n}\right\}$ denotes a set of $n$ alternatives. The evaluation criteria are factors on which alternatives are evaluated and compared. Formally, a criterion is a function $g_{j}$, defined on $A$, taking its values in an ordered set, and representing the DM's preferences according to some points of view. The evaluation of an alternative $x$ according to criterion $g_{j}$ is written $g_{j}(x)$. Let $F=\{1,2, \cdots, m\}$ denotes the set of criteria indices.

To compare alternatives in $A$, we need to aggregate the partial evaluations (i.e. in respect to each criterion) into a global one by using a given aggregation function. Within discrete family, there are usually two aggregation approaches: (i) utility function-based approach, and (ii) outranking relation-based approach. In the rest of this paper we focalize on the second approach. Outranking methods 
use partial aggregation functions. Indeed, criteria are aggregated into partial binary relation $S$, such that $a S b$ means that " $a$ is at least as good as $b$ ". The binary relation $S$ is called outranking relation. The most known method in this family is ELECTRE (see [4]). To construct the outranking relation $S$, we compute for each pair of alternatives $(x, y)$, a concordance indices $C(x, y) \in[0,1]$ measuring the power of criteria that are in favor of the assertion $x S y$ and a discordance indices $N D(x, y) \in[0,1]$ measuring the power of criteria that oppose to $x S y$. Then, the relation $S$ is defined as follows:

$$
\left\{\begin{array}{l}
C(x, y) \geq \hat{c} \\
N D(x, y) \leq \hat{d}
\end{array}\right.
$$

where $\hat{c}$ and $\hat{d}$ and a concordance and a discordance threshold, respectively. Often an exploitation phase is needed to "extract", form $S$, information on how alternatives compare to each other. At this phase, the concordance and discordance indices $(C(x, y)$ and $N D(x, y))$ are used to construct indices $\sigma(x, y) \in[0,1]$ representing the credibility of the proposition $x S y, \forall(x, y) \in A \times A$. The proposition $x S y$ holds if $\sigma(x, y)$ is greater or equal to a given cutting level, $\lambda$.

In spatial contexts, alternatives are often modeled through one of three spatial entities, namely point $(P)$, line $(L)$, or area (or polygon) $(Q)$. For instance, in a facility location problem potential alternatives take the form of points representing different possible sites. This way of modeling generates a rich set of spatial alternatives. As consequence, outranking-based methods quickly reach their computational limits. Evaluation criteria are associated with geographical entities and relationships between entities and therefore can be represented in the form of maps. A criterion map is composed of a collection of spatial units; each of which is characterized with one value relative to the concept modeled. Mathematically, a criterion map $\mathbf{c}_{\mathbf{j}}$ is the set $\left\{\left(s, g_{j}(s)\right): s \in S_{j}\right\}$ where $S_{j}$ is a set of spatial units and $g_{j}$ is a mono-valued criterion function defined as follows:

$$
\begin{aligned}
g_{j}: S_{j} & \rightarrow E \\
s & \rightarrow g_{j}(s)
\end{aligned}
$$

$E$ is an ordinal (or cardinal scale). One should distinguish a simple map layer from a criterion map. In fact, a criterion map models the preferences of the DM concerning a particular concept, which is often with no real existence while a simple map layer is a representation of some spatial real data. In practice, criteria are often of different types and may be evaluated according to different scales. Here we suppose that criteria are evaluated on the same ordinal scale.

\section{Concept of decision map}

\subsection{Definition}

A decision map is a planar subdivision represented as a set of non-overlapping polygonal spatial units that are assigned using a multicriteria sorting model, $\Gamma_{\omega}$, into an ordered set of categories representing evaluation levels. More formally, a decision map $\mathbf{M}$ is defined as $\mathbf{M}=\left\{\left(u, \Gamma_{\omega}(u)\right): u \in U, \omega \in \Omega\right\}$, where $U$ is a set of homogenous spatial units and $\Gamma_{\omega}$ is defined as follows: 


$$
\begin{aligned}
\Gamma_{\omega}: U & \rightarrow E \\
u & \rightarrow \Gamma_{\omega}\left[g_{1}(u), \cdots, g_{m}(u), w\right]
\end{aligned}
$$

where: (i) $E:\left[e_{1}, e_{2}, \cdots, e_{k}\right]$ : (with $e_{i} \succ e_{j}, \forall i>j$ ) is an ordinal scale defined such that $e_{i}$, for $i=1 . . k$, represents the evaluation of category $C_{i}$; (ii) $g_{j}(u)$ : is the performance of spatial unit $u$ in respect to criterion $g_{j}$ associated with criteria map $\mathbf{c}_{j}$; (iii) $\Omega$ : is the space of possible values for preference parameters vector $\tau=\left(\tau_{1}, \tau_{2}, \cdots, \tau_{v}\right)$ associated with $\Gamma_{\omega}$; and (iv) $\omega \in \Omega$ : a single vector of preference parameters values.

Spatial units need to be non-overlapping and together constitue $\mathbf{M}$. Let $I=$ $\{1,2, \cdots, n\}$ be the set of the indices of the spatial units composing M. Then, two conditions need to be verified:

$$
\mathbf{M}=\bigcup_{i \in I} u_{i}, \quad \text { and } \quad u_{i}^{\circ} \cap u_{j}^{\circ}=\emptyset, \forall i, j \in I \wedge i \neq j .
$$

The first condition ensures that the partition is total. The second one ensures that spatial units are non-overlapping. In addition, we generally suppose that the evaluations of connected spatial units are distinct, that is:

$$
\partial u_{i} \cap \partial u_{j} \neq \emptyset \Leftrightarrow \Gamma_{w}\left(u_{i}\right) \neq \Gamma_{w}\left(u_{j}\right), \forall i, j \in I \quad \text { and } \quad i \neq j .
$$

\subsection{Construction of the planar subdivision}

The construction of a decision map needs the superposition of a set of criteria maps. The result is an intermediate map I composed of a new set of spatial units that result from the intersection of the boundaries of the features in criteria maps. Each spatial unit $u$ is characterized with a vector $\mathbf{g}(u)$ of $m$ evaluations:

$$
\begin{aligned}
\mathbf{I} & =\oplus\left(\mathbf{c}_{\mathbf{1}}, \mathbf{c}_{\mathbf{2}}, \cdots, \mathbf{c}_{\mathbf{m}}\right) \\
& =\left\{(u, \mathbf{g}(u)): \mathbf{g}(u)=\left(g_{1}(u), g_{2}(u), \cdots g_{m}(u)\right)\right\} .
\end{aligned}
$$

where $\oplus$ is the union variant of GIS overlay operation that yields a new map by combining all involved features in the input maps; and for $j=1 \cdots m$, $g_{j}(u)$ is the criterion function associated with criterion map $\mathbf{c}_{\mathbf{j}}$. Intuitively, criteria maps must represent the same territory and must be defined according to the same spatial scale and the same coordinate system. Note also that overlay operation may generate silver polygons which should be eliminated. In addition, we mention that criteria maps must be polygonal ones. However, input datasets may be sample data points, raster maps, contour lines lines, etc. We need to transform all non-polygonal input datasets into polygonal ones. For example, a set of sample points may be transformed into a TIN by computing the triangulation having vertices at data points or contour lines may be transformed into a polygonal map by a constrained Delaunay triangulation (see, for e.g., [5]).

The first version of $\mathbf{M}$ is then obtained by applying the multicriteria sorting model $\Gamma_{\omega}$ to associate each spatial unit $u$ in $\mathbf{I}$ to a category in $E$ : 


$$
\begin{aligned}
\mathbf{M}: \mathbf{I} & \longrightarrow E \\
u & \longrightarrow \Gamma_{\omega}(u)
\end{aligned}
$$

The multicriteria sorting model $\Gamma_{\omega}$ used here will be detailed in $\S 4.3$. To generate the final decision map $\mathbf{M}$, we need to group, using Algorithm 1 below, the neighbors spatial units which are assigned to the same category. There are different ways to define the "neighbors" concept. Here, we consider that two spatial units $u_{i}$ and $u_{j}$ are neighbors only and only if they share at least one segment: $\left(\partial u_{i} \cap \partial u_{j} \neq \emptyset\right)=$ true. Other neighboring models may also apply. Note that $v(u)$ in Algorithm 1 denotes the set of "neighbors" of $u$.

Algorithm 1 GROUPING (M)

begin

$u \leftarrow u_{1}$

$Z \leftarrow \emptyset$

While $(\exists u \in \mathbf{I} \wedge u \notin Z)$

For each $s \in v(u)$

If $\Gamma_{\omega}(s)=\Gamma_{\omega}(u)$ Then $\operatorname{MERGE}(u, s)$

End If

End For

$Z \leftarrow Z \cup\{u\}$

End While

end.

MERGE is a map algebra operator permitting to combine two or more spatial units.

\subsection{Multicriteria sorting model}

The multicriteria sorting model $\Gamma_{\omega}$ used is ELECTRE TRI (see [4]). The levels of scale $E$ represents the evaluations of $p$ categories defined in termes of a set of $p-1$ profiles. Let $B=\left\{b_{1}, b_{2}, \cdots, b_{p-1}\right\}$ be the set of indices of the profiles, $b_{h}$ being the upper limit of category $C_{h}$ and the lower limit of category $C_{h+1}$, $h=1,2, \cdots, p$. Each profile $b_{h}$ is characterized by its performances $g_{j}\left(b_{h}\right)$ and its thresholds $p_{j}\left(b_{h}\right)$ (preference thresholds representing, for two alternatives $x$ and $y$, the smallest difference compatible with a preference in favor of $x$ in respect to criterion $\left.g_{j}\right), q_{j}\left(b_{h}\right)$ (indifference thresholds representing, for two alternatives $x$ and $y$, the largest difference preserving an indifference between $x$ and $y$ in respect to criterion $g_{j}$ ) and $v_{j}\left(b_{h}\right)$ (veto thresholds representing, for two alternatives $x$ and $y$, the smallest difference $g_{j}(y)-g_{j}(x)$ incompatible with $\left.x S y\right)$.

The preference parameters vector associated with ELECTRE TRI is $\tau=$ $(\mathbf{k}, \mathbf{q}, \mathbf{p}, \mathbf{v}, \mathbf{B})$, where: (i) $\mathbf{k}=\left(k_{1}, \cdots, k_{m}\right)$ is the weights vector associated with evaluation criteria reflecting their importance for the DM; (ii) $\mathbf{q}=\left[q_{j}\left(b_{h}\right)\right]$, $j \in F, h \in B$ is the indifference thresholds parameters; (iii) $\mathbf{p}=\left[p_{j}\left(b_{h}\right)\right]$, $j \in F, h \in B$ is the preference thresholds parameters; (iv) $\mathbf{v}=\left[v_{j}\left(b_{h}\right)\right], j \in$ $F, h \in B$ is the veto thresholds parameters; and $(\mathrm{v}) \mathbf{B}=\left(\mathbf{b}_{\mathbf{0}}, \mathbf{b}_{\mathbf{1}}, \cdots, \mathbf{b}_{\mathbf{p}}\right)^{T}$ 
is the profiles evaluation matrix with $\mathbf{b}_{\mathbf{h}}=\left(g_{1}\left(b_{h}\right), \cdots, g_{n}\left(b_{h}\right)\right)$. Note that $\mathbf{b}_{\mathbf{0}}$ and $\mathbf{b}_{\mathbf{p}}$ are defined as follows: $\mathbf{b}_{\mathbf{0}}=\left(\min _{u \in U}\left(g_{1}(u), \cdots, \min _{u \in U}\left(g_{m}(u)\right)\right.\right.$ and $\mathbf{b}_{\mathbf{p}}\left(\max _{u \in U}\left(g_{1}(u), \cdots, \max _{u \in U}\left(g_{m}(u)\right)\right.\right.$. It is obvious that different values for $w$ may lead to different assignment results.

ELECTRE TRI has two assignment algorithms: pessimistic and optimistic. Algorithm 2 provides the pessimistic version.

Algorithm 2 ASSIGNMENT $\left(\Gamma_{\omega}(u), \forall u \in \mathbf{I}\right)$

begin

For each $u \in \mathbf{I}$

$h \leftarrow p$

$\mathbf{g}(u) \leftarrow\left(g_{1}(u), \cdots, g_{m}(u)\right)$

assigned $\leftarrow$ False

While $h \geq 0$ and $\neg$ (assigned)

$\mathbf{g}\left(b_{h}\right) \leftarrow\left(g_{1}\left(b_{h}\right), \cdots, g_{m}\left(b_{h}\right)\right)$

$w^{\prime} \leftarrow\left(\mathbf{q}\left(\mathbf{b}_{\mathbf{h}}\right), \mathbf{p}\left(\mathbf{b}_{\mathbf{h}}\right), \mathbf{v}\left(\mathbf{b}_{\mathbf{h}}\right)\right)$

If SIGMA $\left(\mathbf{g}(u), \mathbf{g}\left(b_{h}\right), \mathbf{k}, w^{\prime}\right) \geq \lambda$ Then

$\Gamma_{\omega}(u) \leftarrow e_{h+1}$

assigned $\leftarrow$ True

End If

$h \leftarrow h-1$

End While

End For

end.

The boolean variable assigned is used to avoid unnecessary loops. The algorithm SIGMA permits to compute credibility index $\sigma\left(u, b_{h}\right)$ measuring the degree to which spatial unit $u$ outranks profile $b_{h}: u S b_{h}$. The complexity of SIGMA is $O(m)$; where $m$ is the number of evaluation criteria (see [4] for more information). The parameter $\lambda \in[0.5,1]$ is the cutting level representing the minimum value for $\sigma\left(u, b_{h}\right)$ so that $u S b_{h}$ holds.

\section{$5 \quad$ Generating spatial decision alternatives}

As mentioned earlier, spatial decision alternatives are often modeled through punctual, linear or areal features. This may generate a large set of alternatives which makes outranking methods non practical since they quickly reach their computational limitations. To avoid this problem, we propose in this section different solutions to generate these alternatives based on the decision map concept introduced in $\S 4$. The basic idea of our solutions consists in "emulate" punctual, linear and areal decision alternatives through one or several spatial units with some additional topological relationships.

- Punctual alternative : One spatial unit.

- Linear alternative : A collection of linearly adjacent spatial units.

- Areal alternative : A collection of contiguous spatial units 


\subsection{Generating punctual alternatives}

Punctual alternatives apply essentially to location problems. They may be modeled as individual spatial units. Thus, the set of potential alternatives $A$ is simply the set of spatial units. Theoretically, any spatial unit may serve as an alternative. However, in practice the DM may wish to exclude some specific spatial units from consideration. Let $X \subset U$ be the set of excluded spatial units: $X=\left\{u_{i}^{\prime}: u_{i}^{\prime} \in U\right.$ and that DM states that $\left.u_{i}^{\prime} \notin A\right\}$. Thus, the set of potential alternatives is: $A=\left\{a_{i}: a_{i} \in U \backslash E\right\}$.

\subsection{Generating linear alternatives}

Linear alternatives are often used to model linear infrastructures as highways, pipelines, etc. They may be modeled as a collection of linearly adjacent spatial units. The generation of this type is more complex than the punctual ones. In this paper, these alternatives are generated basing on the connexity graph resulting from the decision map. The connexity graph $G=(U, V)$ is defined such that : $U=\{u: u \in \mathbf{M}\}$ and $V=\left\{\left(u_{i}, u_{j}\right): u_{i}, u_{j} \in U \wedge \partial u_{i} \cap \partial u_{j} \neq \emptyset \wedge u_{i}^{\circ} \cap u_{j}^{\circ}=\emptyset\right\}$. Each vertices $x$ in $G$ is associated with the evaluation $v_{E}(u)$ of the spatial unit $u$ it represents. In practice, the DM may impose that the linear alternative $t$ must pass through some spatial units or avoid some other ones. Let $Y=\{u \in U$ : $\left.(t \cap u=u) \wedge\left(t^{\circ} \cap u^{\circ} \neq \emptyset\right)\right\}$ be the set of spatial units that should be included and $X=\{u \in U:(t \cap u=\emptyset)\}$ be the set of spatial units to be avoided. The conditions in the definition of set $Y$ signify that $(u$, in, $t)$ is true and the one in the definition of $X$ means that $(u$, disjoint, $t)$ is true. Let also $f(t)$ and $t(t)$ denote the start and end spatial units for an alternative $t$. A linear alternative $t$ is defined as follows:

$$
t=\left\{u_{1}, \cdots, u_{q}: u_{i} \in U \backslash X, i=1 . . q\right\}
$$

with: (i) $\mathrm{f}(t)=u_{1}$ and $\mathrm{t}(t)=u_{q}$; (ii) $\left(\partial u_{i} \cap \partial u_{i+1}\right) \neq \emptyset \wedge u_{i}^{\circ} \cap u_{i+1}^{\circ}=\emptyset, \forall i=$ 1... $q-1$; and (iii) $t \cap Y=Y$. The first condition set the origin and end spatial units. The second condition ensures that spatial units in $t$ are linearly adjacent. The last one ensures that all spatial units in set $Y$ are included in $t$. Alternatives are then generated based on $G^{\prime}\left(U \backslash X, V^{\prime}\right)$ with the condition that these alternatives should pass through spatial units $u_{j}, \forall u_{j} \in Y$. To generate alternatives, we apply the following idea. A linear alternative is defined above as a collection of linear adjacent spatial units. Let $\left(v_{E}\left(u_{1}\right), v_{E}\left(u_{2}\right), \cdots, v_{E}\left(u_{q}\right)\right)$ be the set of the evaluations of all spatial units composing $t$. Then, to evaluate an alternative $t$ we need to map a vector of $q$ evaluations to a vector of $k$ evaluations using a transformation rule $\varphi$ :

$$
\begin{aligned}
\varphi: E^{q} & \rightarrow E^{\prime} \\
\left(v_{E}\left(u_{1}\right), v_{E}\left(u_{2}\right), \cdots, v_{E}\left(u_{q}\right)\right) & \rightarrow\left(e_{1}^{\prime}, e_{2}^{\prime}, \cdots, e_{k}^{\prime}\right)
\end{aligned}
$$

where $E^{\prime}:\left[e_{1}^{\prime}, e_{2}^{\prime}, \cdots, e_{k}^{\prime}\right]$ is an ordinal evaluation scale with $e_{1}^{\prime} \prec e_{2}^{\prime} \prec \cdots \prec$ $e_{k}^{\prime}$ ( $E^{\prime}$ can be the same one used in decision map generation). The level $e_{i}^{\prime}$ may 
be the number of nodes $x_{j}$ (i.e. spatial unit $u_{j}$ ) such that $v_{E}\left(x_{j}\right)=e_{i}$, the area of spatial units $u_{j}$ evaluated $e_{i}$, or any other spatial criterion. Before performing the global evaluation, dominated corridors need to be eliminated from consideration. The dominance relation $\triangle$ can not be defined directly on the initial evaluation vector $\left(v_{E}\left(x_{o}\right), \cdots, v_{E}\left(x_{n}\right)\right)$ since alternatives may have different lengths (in terms of the number of spatial units). It can be expressed on the transformed evaluation vector as follows. Let $t$ and $t^{\prime}$ be two linear alternatives with transformed evaluation vectors $\left(r_{1}, r_{2}, \cdots, r_{k}\right)$ and $\left(r_{1}^{\prime}, r_{2}^{\prime}, \cdots, r_{k}^{\prime}\right)$, respectively. Then $t$ dominates $t^{\prime}$, denoted $t \Delta t^{\prime}$, holds only and only if: $t \Delta t^{\prime} \Leftrightarrow r_{i} \succeq r_{i}^{\prime}, \forall i=1 . . k$ with at least one strict inequality. The global evaluation of an alternative $t$ is $v(t)=\boldsymbol{\Theta}\left(r_{1}, r_{2}, \cdots, r_{k}\right)$ where $\boldsymbol{\Theta}$ is an aggregation mechanism.

\subsection{Generating areal alternatives}

In several problems, alternatives are often modeled as a collection of contiguous spatial units. To generate this type of alternatives, we use the following idea. Let $T^{\alpha}=\left\{u_{j} \in U: v_{E}\left(u_{j}\right)=\alpha\right\}$ be the set of spatial units in $U$ with level $\alpha ; \alpha=1 . . k$. Let $T_{i}^{\beta}=\left\{u_{j} \in U: \partial u_{i} \cap \partial u_{j} \neq \emptyset \wedge v_{E}\left(u_{j}\right)=\max _{l \in E \wedge l<\beta} l\right\}$ be the set of spatial units that are contiguous to $u_{i}$ and having the best evaluation strictly inferior to $\alpha$. Next, we construct a tree $T$ defined as follows. To each spatial unit $u_{i}$ in $T^{\alpha}$ associate spatial units in $T_{i}^{\beta} ; \beta \prec \alpha$, as suns. Note that if $\left|T^{k}\right|>1$, we need to create a hypothetic node $r$ having as suns the spatial units in $T^{k}$. Then, an areal alternative $a$ may be constructed as a collection of spatial units in an elementary path starting in a spatial unit in $T^{k}$ (or $r$ if $\left|T^{k}\right|>1$ ) and continues until some conditions (e.g. the total surface of spatial units in the path) are verified.

\section{Implementation}

We have developed a prototype on ArcGIS 9.1 by using VBA. The prototype permits to create criteria maps, infer preference parameters for ELECTRE TRI, assigning spatial units to categories, and generate decision alternatives. We have used real data relative to Ile-De-France (Paris and its suburban) region in France and three illustrative problems of location, corridor generation and zoning have been considered. Here we briefly comment the second problem. Three criteria maps (land-use, sol type and administrative limitations) and four categories have considered. Figure 1 presents two corridors generated using the idea described in $\S 5.2$.

\section{Conclusion and future work}

We have proposed an approach to generate spatial decision alternatives in multicriteria spatial decision making contexts. The proposed approach uses a planar subdivision of the study area, called decision map, composed of a set of nonoverlapping set of spatial units. These spatial units are then used to "emulate" 


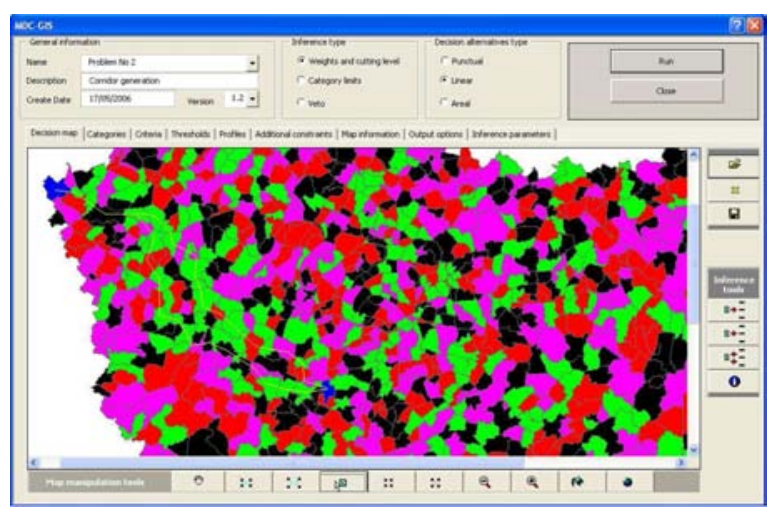

Fig. 1. A screen from the prototype showing two corridors.

punctual, linear and areal decision alternatives often used as input for MCA methods. This way of modeling permits to reduce significantly the number of alternatives to be evaluated enabling outranking methods to be used. The proposed solutions have been implemented on ArcGIS 9.1 by using real data relative to Ile-De-France region in France.

\section{References}

1. Araújo, C., Mecedo A.: Multicriteria geologic data analysis for mineral favourability mapping: application to a metal sulphide mineralized area, Ribeira Valley Metalogenic Province, Brazil", Nat. Res. Resea. 11 (2002) 29-43

2. Clementini, E., Di Felice, P., van Oosterrom, P.: A small set of formal topological relationships suitable for end-user interaction In: proc. 3rd International Symposium on Large Spatial Databases, Lecture Notes in Computer Science, Vol. 1281. SpringerVerlag, Berlin Heidelberg New York (1997) 415-438

3. Clementini, E., Di Felice, P.: A comparison of methods for representing topological relationships, Inf. Sci. 3 (1995) 149-178

4. Figueira, J., Mousseau, V., Roy., B. ELECTRE methods. In: J. Figueira, J, Greco, S, Ehrgott, M (eds.): Multiple criteria decision analysis: State of the art surveys. Springer-Verlag, N.Y. (2005) 133-162

5. de Floriani, L., Magillo, P., Puppo, E.: Applications of computational geometry to geographic information systems. In: Sack, J.-R, Urrutia, J. (eds.): Handbook of Computational Geometry. Elsevier Science B.V. (1999) 333-386

6. F. Joerin, F., Thériault, M., Musy, A.: Using GIS and outranking multicriteria analysis for land-use suitability assessment, Int. J. Geo. Inf. Sci. 15 (2001) 153-174

7. Malczewski, J.: A GIS-based multicriteria decision analysis: a survey of the literature, Int. J. Geo. Inf. Sci. 20 (2006) 703-726

8. Marinoni, O.: A stochastic spatial decision support system based on PROMETHEE, Int. J. Geo. Inf. Sci. 19 (2005) 51-68

9. Marinoni, O.: A discussion on the computational limitations of outranking methods for land-use suitability assessment, Int. J. Geo. Inf. Sci. 20 (2005) 69-87 